\title{
Swabbing Surgical Sites Does Not Improve the Detection of Staphylococcus aureus Carriage in High-Risk Surgical Patients
}

\author{
Jennifer Brown, Chin-Shang Li, ${ }^{2}$ Mauro Giordani, ${ }^{3}$ Kiarash Shahlaie, ${ }^{4}$ Eric O. Klineberg, ${ }^{3}$ \\ Joanna R. Tripet-Diel, Marie S. Ihara, and Stuart H. Cohen ${ }^{1}$
}

\begin{abstract}
Background: A major risk factor for the development of surgical site infections is Staphylococcus aureus carriage. Compared with non-carriers, S. aureus carriers have up to a 14-fold greater risk for post-surgical infections. Pre-operative screening for $S$. aureus carriage is controversial. Yet, targeted screening in high-risk patients or from clinically relevant sites may be beneficial. We aimed to determine whether $S$. aureus detection in high-risk surgical patients would be increased by culturing surgical sites, in addition to the nares, vs. naresonly culturing.

Methods: Adults undergoing pre-operative evaluations in orthopedic and neurosurgical clinics were eligible for participation. For each subject, specimens were collected from the anterior nares and from the proposed surgical site. Samples were inoculated onto methicillin-resistant $S$. aureus-selective chromogenic agar plates and blood agar plates.

Results: Of 150 subjects, $80(53.3 \%)$ were women and $70(46.7 \%)$ men. The mean age was 61 years and 77/150 $(51.3 \%)$ had a BMI $\geq 30$. Culture results were available for $147 / 150$ subjects. Of the 147 surgical site cultures, $54(36.7 \%), 51(34.7 \%)$, and $28(19.0 \%)$ were collected from knee, hip, and lumbar sites, respectively; the remaining $14(9.5 \%)$ were from cervical, thoracic, or infra-clavicular sites. Overall, 35/147 (23.8\%) nasal cultures grew S. aureus; 29/147 (19.7\%) grew methicillin-susceptible S. aureus (MSSA), and 6/147 (4.1\%) grew methicillin-resistant $S$. aureus (MRSA). Only 2/147 (1.4\%) surgical site cultures grew S. aureus; both grew MSSA and MSSA was cultured also from the nasal swabs of these subjects. Using nasal culture + surgical site culture as "true positive," the percentage of additional $S$. aureus carriers detected by the addition of surgical site screening was zero as compared to nasal screening alone.
\end{abstract}

Conclusions: The detection of $S$. aureus carriage in high-risk surgical patients is not improved by swabbing surgical sites in addition to the nares.

S URGICAL SITE INFECTIONS (SSIs) account for $20 \%$ of all healthcare-associated infections [1]. A major risk factor for the development of SSIs is the asymptomatic carriage of Staphylococcus aureus; compared with non-carriers, colonized patients have up to a fourteen-fold greater risk of postsurgical infections [2-4]. Efforts to reduce this risk have driven the practice of screening pre-operative patients for
$S$. aureus carriage $[1,2,5,6]$. The merits of this tactic are debatable; nonetheless, one recent survey found that, either by preference or by state mandate, more than one-third of respondents' institutions perform such screening [7].

The optimal strategy for detecting $S$. aureus carriage in surgical patients is not well-defined. Screening methods commonly involve testing specimens obtained from the anterior

\footnotetext{
${ }^{1}$ Division of Infectious Diseases, Department of Internal Medicine, University of California, Davis Medical Center, Sacramento, California.

${ }^{2}$ Division of Biostatistics, Department of Public Health Sciences, School of Medicine, University of California, Davis, Sacramento, California.

${ }^{3}$ Department of Orthopedic Surgery, University of California, Davis Medical Center, Sacramento, California.

${ }^{4}$ Department of Neurological Surgery, University of California, Davis Medical Center, Sacramento, California.
} 
nares; however, nares-only screening is insensitive and can underestimate the carriage of $S$. aureus by $30 \%-50 \%[8,9]$. The use of multiple-body-site screening strategies (e.g., nares + perineum + throat + axilla) can increase the detection rate to almost 90\% [8,9]. Yet, this approach is inconvenient, costly, and has not been rigorously evaluated in pre-operative patients.

The results of some studies suggest that the most effective $S$. aureus screening strategies are those that use targeted screening in high-risk populations, or that focus on clinically relevant sites $[8,9]$. With this in mind, we aimed to determine whether the detection rate of $S$. aureus in high-risk surgical patients would be increased by screening surgical sites, in addition to the nares, as compared with nares-only screening.

\section{Patients and Methods}

In this single-center, prospective study, adults $>18$ years old who were undergoing pre-operative evaluations in the outpatient orthopedic and neurosurgical clinics at the University of California, Davis Medical Center (UCDMC) were eligible for participation. Patients with active infections, prisoners, pregnant women, and adults who were unable to provide consent were excluded. Subjects were enrolled from April through November 2013. The study was approved by the UCDMC institutional review board and all subjects provided written informed consent.

The following data were collected for each subject: Age, gender, body mass index (BMI), history of $S$. aureus infection, antibiotic exposure within the $90 \mathrm{~d}$ prior to study enrollment, and medical co-morbidities. For each subject, one swab (Transporter Liquid Stuart Culture Swab, HealthLink, Jacksonville, FL) specimen was collected from the anterior nares and one pre-moistened swab (Transporter Liquid Stuart Culture Swab) specimen was collected from the proposed surgical site. The specimens were collected during the patients' pre-operative clinic visit. Specimens were inoculated onto methicillin-resistant S. aureus (MRSA)-selective chromogenic agar plates (Spectra ${ }^{\mathrm{TM}}$ MRSA, Remel, Lenexa, KS) and blood agar plates. All plates were incubated at $30^{\circ} \mathrm{C}$. The MRSA-selective plates were evaluated for the presence of MRSA colonies by the use of reported methods [10]. The blood agar plates were evaluated for the presence of methicillin-susceptible S. aureus (MSSA) colonies at $48 \mathrm{~h}$ with standard microbiological techniques. The data analysis was generated by use of SAS software, version 9.2. (SAS Institute Inc., Cary, NC).

\section{Results}

One hundred fifty-three subjects were initially enrolled in the study; three were excluded from further analysis because they had received antibiotics with activity against $S$. aureus during the $90 \mathrm{~d}$ prior to study enrollment. Of the remaining 150 subjects, $80(53.3 \%)$ were women and $70(46.7 \%)$ were men. Their mean age was 61 years (range 18-88, median 62, SD 12.48) and mean BMI 30.8 (range 18-51, median 30, SD $5.96) ; 77(51.3 \%)$ had a BMI $\geq 30$. Sixty-seven $(44.7 \%)$ of the subjects had hypertension, 21 (14.0\%) had diabetes mellitus, and $11(7.3 \%)$ had coronary artery disease. The surgical procedures for which the subjects were being evaluated were $55(36.7 \%)$ total knee arthroplasty, 52 (34.7\%) total hip arthroplasty, $29(19.3 \%)$ lumbar spine operation, $6(4.0 \%)$ cervical spine operation, $6(4.0 \%)$ deep brain stimulator placement, and $2(1.3 \%)$ thoracic spine operation.

Of the 150 subjects, three were excluded from microbiologic analysis because of specimen processing errors. Surgical site and nares culture results were available for the remaining 147 subjects (Table 1). Of the surgical site swabs, $54(36.7 \%)$ were collected from the knees, 51 (34.7\%) from the hips, 28 $(19.0 \%)$ from the lumbar region, and 14 (9.5\%) from the cervical, thoracic, or infra-clavicular regions. Of the147 subjects, $35(23.8 \%)$ had nares swab cultures that grew S. aureus, 29 (19.7\%) grew MSSA, and six (4.1\%) grew MRSA. In contrast, $S$. aureus grew only from two (1.4\%) of the surgical site swabs; both grew MSSA and these two patients also had MSSA-positive nares specimens. Thus, in 33 patients, nares swabs were positive for $S$. aureus, whereas their surgical site swabs were negative. Univariable logistic regression analysis did not find a statistically significant association

Table 1. Staphylococcus aureus-Positive Specimens by Swab Site

\begin{tabular}{|c|c|c|c|}
\hline \multirow[b]{2}{*}{$\begin{array}{l}\text { Category of surgical patient } \\
\text { and site(s) swabbed }\end{array}$} & \multicolumn{3}{|c|}{ Number (\%) of subjects } \\
\hline & $\begin{array}{l}\text { With MRSA-positive } \\
\text { specimens }\end{array}$ & $\begin{array}{l}\text { With MSSA-positive } \\
\text { specimens }\end{array}$ & $\begin{array}{l}\text { With S. aureus-positive specimens } \\
\quad(M R S A+M S S A \text { combined })\end{array}$ \\
\hline Knee surgery $(n=54)$ & $3 / 54(5.6 \%)$ & $10 / 54(18.5 \%)^{*}$ & $13 / 54(24.1 \%)^{*}$ \\
\hline Nares & $3 / 54(5.6 \%)$ & $10 / 54(18.5 \%)$ & $13 / 54(24.1 \%)$ \\
\hline Knee & $0 / 54\left(\begin{array}{ll}0 & \%\end{array}\right)$ & $1 / 54(1.9 \%)$ & $1 / 54(1.9 \%)$ \\
\hline Hip surgery $(n=51)$ & $1 / 51(2.0 \%)$ & $8 / 51(15.7 \%)$ & 9/ $51(17.6 \%)$ \\
\hline Nares & $1 / 51(2.0 \%)$ & $8 / 51(15.7 \%)$ & $9 / 51(17.6 \%)$ \\
\hline Hip & $0 / 51\left(\begin{array}{ll}0 & \%\end{array}\right)$ & $0 / 51\left(\begin{array}{ll}0 & \%\end{array}\right)$ & $0 / 51\left(\begin{array}{ll}0 & \%\end{array}\right)$ \\
\hline Lumbar surgery $(\mathrm{n}=28)$ & $1 / 28(3.6 \%)$ & $8 / 28(28.6 \%)$ & 9/ $28(32.1 \%)$ \\
\hline Nares & $1 / 28(3.6 \%)$ & $8 / 28(28.6 \%)$ & $9 / 28(32.1 \%)$ \\
\hline Lumbar & $0 / 28(0 \%)$ & $0 / 28(0 \%)$ & $0 / 28(0 \%)$ \\
\hline $\begin{array}{l}\text { Cervical, thoracic or infra-clavicular } \\
\text { surgery }(\mathrm{n}=14)\end{array}$ & $1 / 14(7.1 \%)$ & $3 / 14(21.4 \%)^{*}$ & $4 / 14(28.6 \%)^{*}$ \\
\hline Nares & $1 / 14(7.1 \%)$ & $3 / 14(21.4 \%)$ & $4 / 14(28.6 \%)$ \\
\hline Cervical, thoracic or infra-clavicular & $0 / 14(0 \%)$ & $1 / 14(7.1 \%)$ & $1 / 14(7.1 \%)$ \\
\hline Total $(n=147)$ & $6 / 147(4.1 \%)$ & $29(19.7 \%)$ & $35 / 147(23.8 \%)$ \\
\hline
\end{tabular}

*The patients with positive surgical site cultures also had positive nares cultures.

MRSA = Methicillin-resistant Staphylococcus aureus; MSSA: Methicillin-susceptible Staphylococcus aureus. 
between $S$. aureus-positive specimens and gender, coronary artery disease, hypertension, diabetes mellitus, or BMI. Using the combined results for MRSA and MSSA, and using nares culture + surgical site culture as an approximation of "'true positive," we found that the percentage of additional $S$. aureus carriers detected by the addition of surgical site screening was zero as compared with nares screening alone.

\section{Discussion}

The results of the present study indicate that the detection of $S$. aureus carriage in high-risk orthopedic and neurological surgical patients is not improved by screening surgical sites in addition to the nares. It may be that screening other surgical sites, such as the sternum in cardiothoracic surgery patients or the inguinal region in hernia surgery patients, would detect higher rates of $S$. aureus carriage than those detected from the surgical sites examined in this study. Also, is possible that the rate of $S$. aureus detection in surgical patients could be improved with the use of more sensitive nucleic acid-based testing methods and/or extensive multiple-body-site screening strategies. However, these approaches are more cumbersome and resource-intensive. Moreover, such intensive, pre-operative screening strategies would not prevent SSIs that result from the intra-operative or postoperative de novo acquisition of $S$. aureus.

As health care institutions strive to reduce SSIs, it is important to focus on the most effective and efficient infection prevention strategies. The results of this study suggest that continued emphasis on proven measures, such as antimicrobial prophylaxis, surgical team antisepsis, and operating room asepsis are better approaches to SSI prevention than screening surgical sites for $S$. aureus carriage.

In summary, we found that the detection of $S$. aureus carriage in high-risk orthopedic or neurological surgery patients is not improved by screening surgical sites in addition to the nares.

\section{Acknowledgments}

We thank the study participants, the surgical clinic personnel, and the UCDMC microbiology laboratory for their assistance with this study.

\section{Author Disclosure Statement}

All authors report no conflicts of interest relevant to this article.

The study was funded by the University of California, Davis Medical Center. J.B. received salary support from the University of California, Davis Medical Center. The study was supported also by the National Center for Advancing Translational Sciences (NCATS), National Institutes of Health (NIH), through grant \#UL1 TR000002.

\section{References}

1. Anderson DJ, Podgorny K, Berríos-Torres SI, et al. Strategies to prevent surgical site infections in acute care hospitals: 2014 update. Infect Control Hosp Epidemiol 2014; 35:605-627.

2. Bratzler DW, Dellinger EP, Olsen KM, et al. Clinical practice guidelines for antimicrobial prophylaxis in surgery. Surg Infect 2013;14:73-156.

3. Kalmeijer MD, van Nieuwland-Bollen E, Bogaers-Hofman $\mathrm{D}$, et al. Nasal carriage of Staphylococcus aureus is a major risk factor for surgical-site infections in orthopedic surgery. Infect Control Hosp Epidemiol 2000;21:319-323.

4. Kluytmans JA, Mouton JW, Ijzerman EP, et al. Nasal carriage of Staphylococcus aureus as a major risk factor for wound infections after cardiac surgery. J Infect Dis 1995; 171:216-219.

5. Schweizer M, Perencevich E, McDanel J, et al. Effectiveness of a bundled intervention of decolonization and prophylaxis to decrease Gram positive surgical site infections after cardiac or orthopedic surgery: Systematic review and meta-analysis. BMJ 2013;346:f2743.

6. van Rijen M, Bonten M, Wenzel R, et al. Mupirocin ointment for preventing Staphylococcus aureus infections in nasal carriers. Cochrane Database Syst Rev 2008;(4): CD006216.

7. Kline S, Highness M, Herwaldt LA, et al. Variable screening and decolonization protocols for Staphylococcus aureus carriage prior to surgical procedures. Infect Control Hosp Epidemiol 2014;35:880-882.

8. Matheson A, Christie P, Stari T, et al. Nasal swab screening for methicillin-resistant Staphylococcus aureus-how well does it perform? A cross-sectional study. Infect Control Hosp Epidemiol 2012;33:803-808.

9. McKinnell JA, Huang SS, Eells SJ, et al. Quantifying the impact of extranasal testing of body sites for methicillinresistant Staphylococcus aureus colonization at the time of hospital or intensive care unit admission. Infect Control Hosp Epidemiol 2013;34:161-170.

10. Peterson JF, Riebe KM, Hall GS, et al. Spectra MRSA, a new chromogenic agar medium to screen for methicillinresistant Staphylococcus aureus. J Clin Microbiol 2010; 48:215-219.

Address Correspondence to: Dr. Jennifer Brown Division of Infectious Diseases Department of Internal Medicine University of California, Davis Medical Center 4150 V. St., PSSB-G500 Sacramento, CA 95817

E-mail: jennifer.brown@ucdmc.ucdavis.edu 